\title{
Novel techniques in high speed modem implementation using programmable DSPs
}

\author{
G N S KUMAR and K HARINATH REDDY \\ Signion Systems Pvt. Ltd 6-3-569/1/2, Rockdale Compound, Somajiguda, \\ Hyderabad, India 500482
}

\begin{abstract}
We present the design and performance of a high speed MSK modem that incorporates digital heterodyne processing. A novel table look-up procedure for direct IF/RF modulation, based on a suitable maximal-length shift register sequence, is described. High performance, linear phase, lowcomplexity mixing and filtering in the receiver is achieved using multirate IFIR filters, where the shaping filter may possibly be $M$ th-band. The configuration of the IFIR filters is chosen based on the carrier frequency and the filtering requirements.
\end{abstract}

Keywords. Minimum shift keying; direct IF/RF modulation; multirate IFIR filters.

\section{Introduction}

There is an ever increasing demand for low cost, high speed data communication systems in a variety of applications such as VSATs, PCS, radio paging, utility metering etc. In this paper, we present design and implementation details of a Minimum Shift Keying (MSK) modem using a novel transmitter and receiver structure. The features and architecture of modern low-cost DSP chips are exploited by the techniques presented herein. Our algorithms implemented on a modern low-cost 33 MIPS processor allow data rates upto $384 \mathrm{kbps}$ and carriers of upto $1 \mathrm{MHz}$.

In recent years MSK (Pasupathy 1979; Austin et al 1983) and its variants (in general, Continuous Phase Frequency Shift Keying - CPFSK) have become increasingly popular modulation techniques for signaling through bandwidth and amplitude limited channels in which data must be efficiently packed into a restricted bandwidth. In the case of MSK, $99 \%$ of the signal power is contained in a bandwidth of about 1.2R (where $R$ is the bit rate) as compared to 8.0R for QPSK and OQPSK. It is also easier to filter an SK signal because of relatively smaller side lobes. The advantage of MSK over QPSK is its phase continuity. As a result, the inter-symbol interference caused by nonlinear amplifiers or hard limiting in satellite applications may be avoided. Further, MSK allows use of efficient digital IFIR filtering, which in turn reduces the complexity of analog filters used in RF sections.

This paper presents a novel technique for direct IF/RF MSK modulation using a look-up table generated from a maximal length shift register. It is shown that the complexity of the 


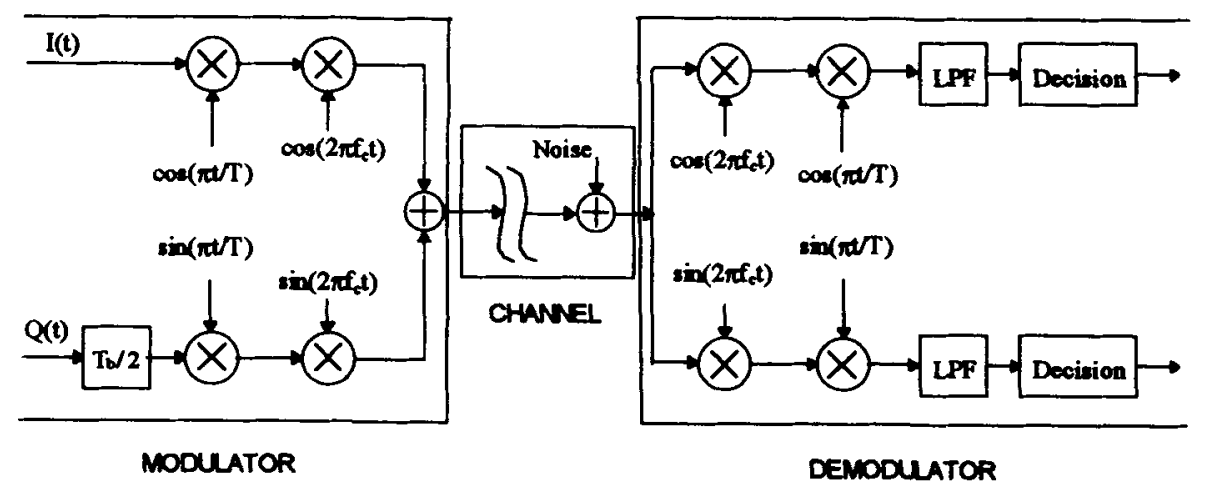

Figure 1. Block diagram of MSK modem.

modulator decreases with increase in the number of bits taken for modulation at a time, $N$, at the expense of memory. Choosing $N$ as even reduces the complexity of the modulator as the start phase has only two states for every $N$ bit input. This look-up table scheme ${ }^{1}$ can further be generalized to GMSK modulation (Jayasimha \& Harinath Reddy 1995) used in GSM and DECT. Multirate IFIR filtering techniques (Neuvo et al 1984) are exploited for low complexity heterodyne processing in the receiver.

Section 2 of this paper provides definition of terms and describes the modern architecture. Section 3 provides implementation details of the IF/RF MSK modulator using look-up tables, heterodyne processing using multirate IFIR filters, digital matched filter and synchronization processing. Section 4 provides performance results of the modem in the presence of additive Gaussian distributed random noise.

\section{Modem configuration}

A block diagram of the modem is as shown in figure 1. In MSK, the in-phase (I) and quadrature-phase $(\mathrm{Q})$ data streams are skewed by $T / 2$ duration in the modulator and are half-cycle sinusoidally weighted (Austin et al 1983).

The mathematical expression for the output of the MSK transmitter is (Pasupathy 1979)

$$
S(t)=\left[a_{I}(t) \cos (\pi t / 2 T) \cos \left(2 \pi f_{c} t\right)+a_{Q}(t) \sin (\pi t / 2 T) \sin \left(2 \pi f_{c} t\right)\right]
$$

where $T$ is the bit duration and $f_{c}$ is the carrier frequency.

The above equation can also be written as

$$
S(t)=\cos \left[2 \pi f_{c} t+b_{k}(t)(\pi t / 2 T)+\phi_{k}\right] .
$$

Here $b_{k}$ is +1 , if $a_{I}$ and $a_{Q}$ have opposite signs and is -1 , if $a_{I}$ and $a_{Q}$ have the same sign. $\phi_{k}$ is 0 or $\pi$ corresponding to $a_{I}=1$ or -1 . Note that $b_{k}(t)$ can also be written as $\left[-a_{I}(t) \cdot a_{Q}(t)\right]$.

The signaling frequencies in MSK from (2) are $f_{h}=\left(f_{c}+f_{b} / 4\right)$ and $f_{l}=\left(f_{c}-f_{b} / 4\right)$. Hence the frequency deviation is equal to half the bit rate, i.e., $\left(f_{h}-f_{l}\right)=f_{b} / 2$. This is the minimum frequency spacing that allows the two FSK signals to be coherently orthogonal, hence the name "Minimum Shift Keying".

\footnotetext{
${ }^{1}$ The design of a look-up table using maximal length shift register was suggested by $\mathrm{S}$ Jayasimha
} 
The carrier phase is given by $\theta(t)=\left[b_{k}(t)(\pi t / 2 T)\right]= \pm(\pi t / 2 T)$. This increases or decreases linearly during each bit period of $T$ seconds. A bit $b_{k}$ of +1 corresponds to an increase of the carrier phase by $90^{\circ}$ and corresponds to a higher frequency $f_{h}$. Similarly $b_{k}=-1$ implies a linear decrease of phase by $90^{\circ}$ over $T$ seconds, corresponding to the lower frequency $f_{l}$. In order to make phase continuous at bit transitions, the carrier frequency $f_{c}$ should be chosen such that $f_{c}$ is an integral multiple of $f_{b} / 4, f_{b}$ being the bit rate and $f_{c}$ the carrier frequency.

An optimum receiver should use a matched filter which delivers sufficient statistics for the decision device. The matched filter receiver consists of correlating the received MSK signal with the recovered carrier and a sinusoid at half the symbol rate followed by an integration operation.

\section{Digital implementation of high speed modem}

The implementation of a high speed modem on a single DSP depends on the use of memory and computationally efficient algorithms for modulation and demodulation.

In general, MSK modulation at the desired carrier can be implemented on a DSP in two ways:

1. The use of bit-by-bit algorithm in which individual input bits change the state of the modulated waveform between higher and lower frequency $f_{h}$ and $f_{l}$, preserving the phase continuity across bauds.

2. The use of a table look-up scheme to modulate multiple bits at once.

However, a brute force look-up table modulator would use a large amount of memory, which works out to $\left[N \cdot \alpha \cdot 2^{N+1}\right]$ locations of memory, where $N$ is the number of bits taken for modulation at a time and is the number of samples per bit. An innovative approach, which minimizes the look-up table memory required is described in the following subsection.

\subsection{High speed MSK modulator}

The modulator employs two look-up tables - Wave table and Index table. The Wave table stores coded MSK waveforms with a suitable IF carrier in the ordering obtained from a linear shift register sequence. Note that the wave table generated here is applicable only for carrier frequency which is an integral multiple of $f_{b} / 4$.

Consider a case of modulating $N$ input bits at a time. Here, $N$ is chosen as an even number to ensure that the modulated waveform corresponding to the $N$ bits end with a phase of either 0 or $\pi$. The output sequence from the shift register contains a start phase bit for the next $N$ input bits along with 2 new bits. It should be noted that the complexity of modulator decreases with increase in $N$.

For example, let $N=4$. Given $P_{n}(x)$ is a polynomial of degree 4 , the following recurrence relation is used to generate a sequence of polynomials:

$$
P_{n+1}(x)=\left[x^{2} \cdot P_{n}(x)+x^{4}+x^{7} \cdot P_{n}(x)\right]
$$

where

$$
\begin{aligned}
& x^{9}=1 \\
& x^{6}=x^{4}+x \\
& x^{5}=x^{4}+x+1 \\
& x^{k}=0 \quad \text { except for } \quad k=0,6,9 .
\end{aligned}
$$


The coefficient $x^{k}$ of the polynomial denotes the starting phase: 0 corresponds to a starting phase of 0 and 1 to a starting phase of $\pi$. The remaining bits correspond to the frequencies of the waveform, for $k$ bit durations, where a 0 denotes the low frequency, $f_{l}$, and 1 the high frequency $f_{h}$. Note that this particular coding corresponds to an implicit differential encoding at the modulator.

The linear recurrence in (3) produces all possible $N$-bit sequences except one, the identity element of the recurrence. In constructing the wave table, the waveform corresponding to the recurrence identity is stored first followed by a waveform that is a continuation of the identity. Thereafter, the recurrence is used to generate the remaining $\left(2^{N+1}-2\right)$ waveforms.

The total size of the table is $\left[2 \cdot \alpha \cdot\left(2^{N+1}+1\right)\right]$ as compared to the brute force look-up table of $\left[N \cdot \alpha \cdot 2^{N+1}\right]$ where $\alpha$ is the number of samples per bit. Other sequences (derived from recurrances or a tree-search procedure) can be used to generate wave tables for other CPM modulation schemes (Jayasimha \& Harinath Reddy 1995).

The Index table comprises $2^{N+1}$ start indices to the wave table of the MSK modulated waveform corresponding to the $N$ bit input. It also provides the start phase information for the next $N$ bit input sequence. The table is of length $2^{N+1}$ because the previous phase must prefix the $N$ bit information in order to preserve the phase continuity across bands.

The modulator takes $N$ bits at a time from the incoming bit stream and forms an index with the current phase. This is used to obtain the wave table offset and start phase information for the next $N$ input bits from the Index table. The wave table offset gives the starting address of the MSK modulated waveform corresponding to the $N$ bit input. Starting from this offset in the Wave table, $N$ times the number of samples are transmitted.

\subsection{High speed MSK demodulator}

The demodulator comprises IFIR digital mixing, $I / Q$ separation, matched filtering and synchronization processing.

The received MSK waveform is first separated into in-phase (I) and quadrature-phase $(\mathrm{Q})$ channels using multirate IFIR filtering techniques (Jayasimha \& Harinath Reddy 1995). Matched filtering is then performed on these base-band signals. The matched filter outputs are then rotated by $-\pi / 2$ to account for the linear phase rotation of the MSK waveform. The rotated matched filter outputs are integrated over a baud to estimate the phase. Upon estimating the phase, a test is performed to check whether the signal is present or not. If the signal is present then coherent detection and differential decoding is performed. The bits obtained are then packed into the required format. The following subsections deal with these blocks individually.

3.2a IFIR digital mixer: The digital mixer uses interpolated FIR (IFIR) filters first described by Neuvo et al (1984). The complexity of IFIR filtering can be further reduced by using an equiripple $M t h$-band filter for the shaping filter. This filter was designed using the method described by Jayasimha \& Narasimha Rao (1995). This design produces a low complexity IF/RF digital filter, thereby reducing the order of analog filters used in RF sections. The configuration IFIR filters is chosen based on the carrier frequency and the filtering requirements. In full duplex systems, the transmit and receive frequencies are chosen as $f_{S} / 4$ and $f_{S} / 8$ or vice versa.

The computational advantage of IFIR mixing, downsampling and matched filtering (at 1.5 complex samples per bit) as opposed to IF matched filtering ( 24 real samples per bit) 
Table 1. Comparison of MIPS required at $288 \mathrm{kbps}$.

\begin{tabular}{lcc}
\hline Function & $\begin{array}{c}\text { No heterodyne } \\
\text { processing (MIPS) }\end{array}$ & $\begin{array}{c}\text { With heterodyne } \\
\text { processing (MIPS) }\end{array}$ \\
\hline Digital mixing & nil & 31 \\
Matched filter & 84.6 & 6.33 \\
Sync. processing, & 7.2 & 7.2 \\
Phase estimation \& & & \\
Coherent detection & & \\
\hline Total & 91.80 & 44.5 \\
\hline
\end{tabular}

is clear from table 1, which shows a comparison of the required MIPS at $288 \mathrm{kbps}$ (with three timing hypotheses).

3.2b Digital matched filter: A filter whose impulse response is a time-reversed and delayed version of some signal is said to be matched to that signal. The purpose of the matched filter is to increase the signal component and decrease the noise component at the same time. This is equivalent to maximizing the signal to noise ratio at the output at some instant. The term base-band is used to designate the band of frequencies representing the original signal as delivered by a source of information.

The digital matched filters operating on the base-band signal are:

$$
\begin{aligned}
& X_{k}(\sigma)=\sum_{m=(4 k+\sigma-4) M / 8}^{(4 k+\sigma+4) M / 8} x_{m} \cdot \cos \left(\frac{m}{M}-\frac{k}{2}-\frac{\sigma}{8}\right) \cdot \pi \\
& Y_{k}(\sigma)=\sum_{m=(4 k+\sigma-4) M / 8}^{(4 k+\sigma+4) M / 8} y_{m} \cdot \cos \left(\frac{m}{M}-\frac{k}{2}-\frac{\sigma}{8}\right) \cdot \pi
\end{aligned}
$$

where $M=2$ (bit duration/sample duration) and $\sigma=$ timing offset.

These are finite sum approximations to the exact matched filters. Notice that the range of integration of $X_{k}$ overlaps that of $X_{k+1}$, and similarly for $Y_{k}$ and $Y_{k+1}$. This means that the matched filtering must be accomplished by four matched filters or correlators, one pair for even values of $k$ and the other pair for odd values of $k$.

In order to address the issue of synchronization, a timing offset $\sigma$ has been introduced.

Bit rotation: Because matched filter outputs for successive bits are rotated by $-\pi / 2$, we define

$$
R_{4 m+\mu}(\sigma)+j S_{4 m+\mu}(\sigma)=\left[X_{4 m+\mu}(\sigma)+j Y_{4 m+\mu}(\sigma)\right] \cdot e^{j \mu \pi / 2}
$$

where $\mu=\{0,1,2,3\}$.

This is equivalent to the following re-ordering of filter outputs:

$$
\begin{array}{cccccc}
k & 1 & 2 & 3 & 4 & 5 \\
R_{k} & X_{1} & -Y_{2} & -X_{3} & Y_{4} & X_{5} \\
S_{k} & Y_{1} & X_{2} & -Y_{3} & -X_{4} & Y_{5}
\end{array}
$$


3.2c Synchronization processing: This consists of phase estimation at each timing offset, selection of timing offset and coherent demodulation using the phase estimated at the selected timing offset.

3.2.c (i) Phase estimation - Phase estimation is done by integrating the rotated matched filter outputs over a baud using the following equations.

$$
\begin{aligned}
& R_{s q}(\sigma)=\sum_{k} R_{k}^{2}(\sigma), \\
& S_{s q}(\sigma)=\sum_{k} S_{k}^{2}(\sigma), \\
& R S(\sigma)=\sum_{k} R_{k}(\sigma) \cdot S_{k}(\sigma) .
\end{aligned}
$$

Carrier phases $\theta(\sigma)$ for each of the three timing hypotheses are estimated using:

$$
\theta(\sigma)=0.5 \cdot \tan ^{-1}[N(\sigma) / D(\sigma)]
$$

where

$$
\begin{aligned}
& N(\sigma)=[2 \cdot R S(\sigma)] \text { and } \\
& D(\sigma)=\left[R_{s q}(\sigma)-S_{s q}(\sigma)\right] .
\end{aligned}
$$

The input to the matched filter with $\phi$ as block phase can be given as

$$
I(t)=A \cdot \cos (\pi t / 2 T+\phi) \text { and } Q(t)=A \cdot \sin (\pi t / 2 T+\phi) .
$$

In matched filtering, the incoming signal is multiplied by $\sin (\pi t / 2 T)$ and integrated over a baud, which is given by

$$
\begin{aligned}
& R(t)=(A / 2) \sum[\sin (\pi t / T+\phi)-\sin (\phi)] \\
& S(t)=(A / 2) \sum[\cos (\pi t / T+\phi)+\cos (\phi)] .
\end{aligned}
$$

Then, $[R(t) \cdot S(t)]$ for $n$ bauds is given by

$$
[-A \cdot(n / 2) \cdot \sin (\phi)] \cdot[A \cdot(n / 2) \cdot \cos (\phi)]=\left[\left(A^{2} n^{2} / 8\right) \cdot \sin (2 \phi)\right]
$$

and

$$
\left.\left[R^{2}(t)-S^{2}(t)\right]=\left(A^{2} n^{2} / 8\right) \cdot \cos (2 \phi)\right] .
$$

Now from (10) and (11), we have

$$
\frac{[2 \cdot R(t) \cdot S(t)]}{\left[R^{2}(t)-S^{2}(t)\right]}=\frac{\left(A^{2} n^{2} / 4\right) \cdot \sin (2 \phi)}{\left(A^{2} n^{2} / 4\right) \cdot \cos (2 \phi)}=\tan (2 \phi) .
$$

Hence, the phase $\phi$ can be found from

$$
\phi=\left\{0.5 \cdot \tan ^{-1}\left[\frac{2 . R(t) \cdot S(t)}{R^{2}(t)-S^{2}(t)}\right]\right\} .
$$

3.2c (ii) Signal presence detection - Signal presence detection is implemented using the following relations:

$$
\begin{aligned}
I_{s q}(\sigma) & =R_{s q}(\sigma) \cos ^{2} \theta+S_{s q}(\sigma) \sin ^{2} \theta+2 R S(\sigma) \cos \theta \sin \theta \\
Q_{s q}(\sigma) & =R_{s q}(\sigma) \cos ^{2} \theta+S_{s q}(\sigma) \sin ^{2} \theta-2 R S(\sigma) \cos \theta \sin \theta
\end{aligned}
$$




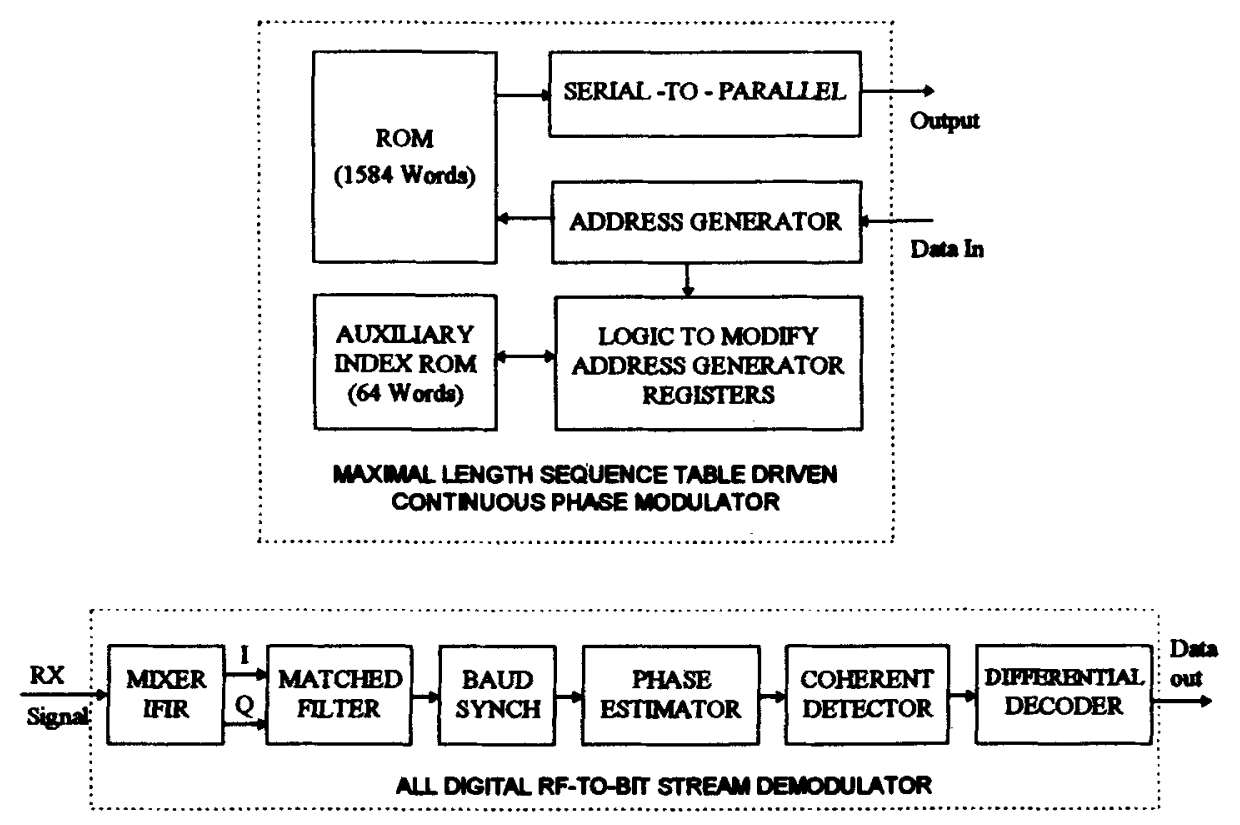

Figure 2. Digital implementation of MSK modem.

The signal is said be present in the following two cases:

1. If $Q_{s q}\left(\sigma_{0}\right)$ is less than threshold $\tau$ times $I_{s q}\left(\sigma_{0}\right)$, then the estimated phase is $\theta\left(\sigma_{0}\right)$ with a timing offset of $\sigma_{0}$.

2. If $I_{s q}\left(\sigma_{0}\right)$ is less than $\tau$ threshold times $Q_{s q}\left(\sigma_{0}\right)$, then the estimated phase is $\left[\theta\left(\sigma_{0}\right)-\right.$ $\pi / 2$ ] with a timing offset of $\sigma_{0}$.

If none of the above two is satisfied, then the signal is said to be not present.

Here, $\tau=0.6612$ is a threshold that gives approximately $10^{-3}$ probability of false alarm in three independent tries at synchronization.

3.2 c(iii) Coherent detection - The decision variables, $T_{k}$, are formed by

$$
T_{k}=\cos \theta \cdot R_{k}\left(\sigma_{0}\right)+\sin \theta \cdot S_{k}\left(\sigma_{0}\right)
$$

The hard decision decoding is done by decoding the decision variable $T_{k}$ as ' 0 ' when it is less than ' 1 ' and as ' 1 ' when it is greater than or equals one.

3.2c(iv) Differential decoder - The bits obtained from the decision variables in coherent detection are passed through a differential decoder of the form,

$$
b_{k}=\left[b_{k} \oplus b_{k-1}\right]
$$

where $\oplus$ stands for modulo- 2 addition. The differential decoded bits thus obtained are packed into the required format.

3.2d Carrier acquisition and tracking: Carrier frequency offsets upto $\left(f_{b} / 128\right) \mathrm{Hz}$ can be acquired by differencing phase estimates over shorter blocks of 16 bits, where $f_{b}$ is the data rate.

Similarly, carrier tracking is done by computing the difference in phase estimates of successive blocks and then applying correction to the carrier accordingly. The frequency range 


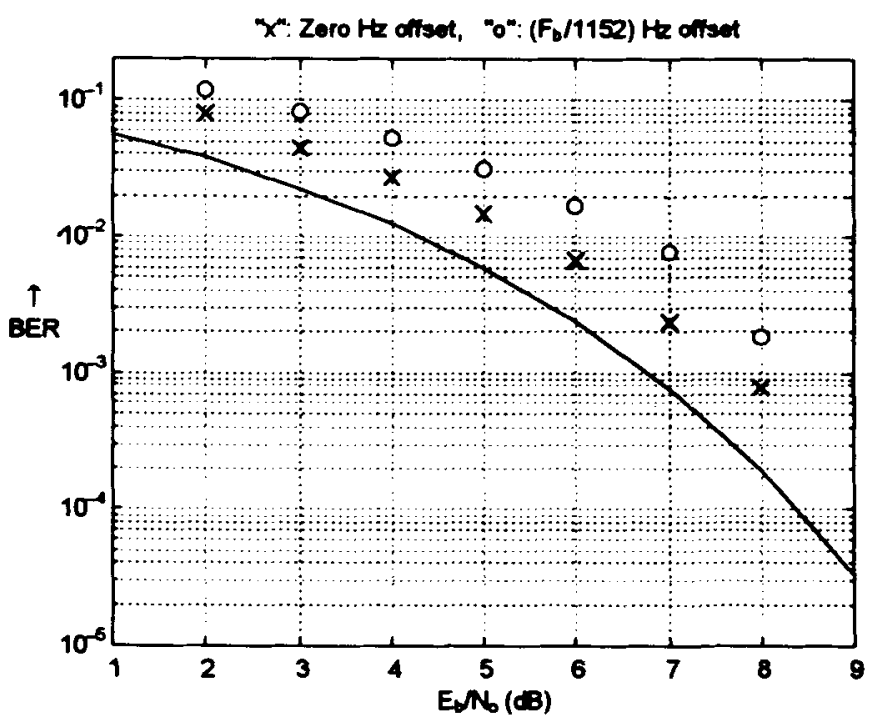

Figure 3. Performance of the modem in AWGN.

over which the demodulator provides accurate frequency offset estimates is $\left(f_{b} / 1152\right) \mathrm{Hz}$ and is the carrier tracking range for block lengths of 144 bits.

The block schematic of modem implemented is as shown in figure 2.

\section{Performance results}

The error probability in BPSK, QPSK and MSK is the same and is given by

$$
P_{e}=0.5 \cdot \operatorname{erf} c \sqrt{E_{b} / N_{o}} \text {. }
$$

This gives the theoretical bit error rate performance which can be compared with the results from the DSP simulations at different noise levels.

By simulation in AWGN, the optimum block size for the phase estimation and timing recovery was determined to be 144 bits. The BER performance of the High Speed MSK modem with IF processing in AWGN is shown in figure 3, where the solid line corresponds to the theoretical curve. Results from the figure indicate that at $\left[E_{b} / N_{0}\right]>7 \mathrm{~dB}$, the performance of the modem is witun $1.0 \mathrm{~dB}$ of the theoretical performance achievable in AWGN.

In the presence of frequency offset, the modem performs well when the phase change over a phase estimate block is less than $\pi / 4$ radians, which corresponds to $41.5 \mathrm{~Hz}$ for 144 bits at a bit rate of $48 \mathrm{kbps}$. The performance of this modem under a frequency offset of $41.5 \mathrm{~Hz}$ is also shown in figure 3 : the performance degradation is less than $0.75 \mathrm{~dB}$ compared to the one with no frequency offset.

\section{Conclusions and summary}

An all-digital implementation of a High Speed Modem using a 33 MIPS digital signal processor and its performance is presented in this paper.

It is shown that the complexity of the modulator decreases with increase in $N$ at the expense of memory. Choosing $N$ as even reduces the complexity of the modulator as the 
start phase is either 0 or $\pi$ for every $N$ bit input. It is also observed from simulations that the optimum block size for phase estimation and bit timing recovery is 144 bits. It is shown that at $\left[E_{b} / N_{0}\right]>7 \mathrm{~dB}$, the performance of the modem with IF processing is within $1.0 \mathrm{~dB}$ of the theoretical performance achievable in AWGN. With a frequency offset of $41.5 \mathrm{~Hz}$, the performance degrades by less than $0.75 \mathrm{~dB}$ compared to the one with no frequency offset.

\section{References}

Austin M C, Ming U C, Daniel F H, Robert A M 1983 QPSK, staggered QPSK, and MSK - A comparative evaluation. IEEE Trans. Commun. COM-31: 171-182

Jayasimha S, Harinath Reddy K 1995 New programmable high speed continuous phase modulator. Proc. SPCOM-95 (IISc, Bangalore), pp. 17-21

Jayasimha S, Narasimha Rao P V R 1995 An iteration scheme for the design of equiripple Mth band filters. IEEE Trans. Signal Process. 43: 1998-2002

Neuvo Y, Dong C Y, Mitra S K 1984 Interpolated finite impulse response filters. IEEE Trans. Acoustics, Speech Signal Process. ASSP-32: 362-370

Pasupathy S 1979 Minimum shift keying: A spectrally efficient modulation. IEEE Commun. Mag. Jul: 14-22 